\title{
Características psicopáticas en la adolescencia: sistematización teórica
}

\section{Psychopathic Features in Adolescence: Theoric Systematization}

Recibido: enero 20 de 2012 | Revisado: marzo 15 de 2012 | Aceptado: mayo 5 de 2012

\author{
ELIZABETH LEÓN-MAYER * \\ DANIELA P. ZÚÑIGA ** \\ Universidad Nacional de La Plata, Buenos Aires, Argentina
}

SICI: 2011-2277(201212)11:4<1197:CPEAST>2.0.CO;2-Z

Para citar este artículo: León-Mayer, E. \& Zúñiga, D. P. (2012). Características psicopáticas en la adolescencia: sistematización teórica. Universitas Psychologica, 11(4), 1197-1207.

Licenciada en Psicología. Magíster en Psicología Social con mención en Psicología Jurídica. Docente UNLP.E-mail: ely_psi@vtr.net. ResearcherID: León-Mayer, E. G-8504-2012.

*** Licenciada en Psicología. Magíster en Psicología Jurídica y Forense. E-mail: dzunigasilva@gmail. com. ResearcherID: Zúñiga, D. G-9042-2012.

\section{RESUMEN}

Hoy existe un interés creciente en estudiar el constructo de psicopatía y su aplicabilidad en población adolescente. La Psychopathy Checklist - Youth Version (PCL-YV) de Forth, Kosson y Hare (2003) deriva de la PCL-R considerando las características propias de la juventud (Zúñiga, 2008). El presente artículo tiene como objetivo dar a conocer tres estudios que constituyen un aporte a la evaluación de características psicopáticas en la juventud. Uno de ellos caracteriza psicométricamentre la PCL-YV aplicada en población chilena (Zúñiga, Vinet \& León, 2011). Otros dos estudios permiten identificar importantes factores de riesgo presentes en la juventud que diferencian a adultos psicopáticos y no psicopáticos (León, Asún \& Folino, 2010; León $\&$ Folino, 2011). Dichas investigaciones permiten sumar conocimientos especializados en el área infantojuvenil.

Palabras clave autores

Psicopatía, evaluación, adolescentes, factores de riesgo.

Palabras clave descriptores

Investigación cuantitativa, psicometría, Chile.

\section{A B S T R A C T}

Nowadays there is a growing interest in the construct of psychopathy and its application to research on youth. The Psychopathy Checklist - Youth Version (PCL-YV) (Forth, Kosson \& Hare, 2003) is a derivative from the PCL-R that includes consideration on the particular characteristics of youngsters (Zúñiga, 2008). This article is a review of three studies that contributed to this area. One is a psychometric characterization of the PCL: YV in Chilean population (Zúñiga, Vinet \& León, 2011). The others, allow the identification of important risk factors present during youth that have different impact in psychopathic and non-psychopathic adults (León, Asún $\&$ Folino, 2010; León \& Folino, 2011). The studies are a contribution to knowledge in this field.

Key words authors

Psychopathy, assessment, adolescents, risk factors.

Key words plus

Quantitative Research, Psychometry, Chile. 


\section{Introducción}

En Chile, durante el año 2009 fueron detenidos 48.902 menores de edad, lo que representa un porcentaje del $9.3 \%$ del total de los detenidos ese año (Blanco, 2010). Esta cifra constituye una gran preocupación para el país, y exige poner en marcha procedimientos especializados que faciliten la comprensión de este tipo de conducta en sujetos que están en proceso de autoformación y de vida en sociedad. Así mismo, se hace necesario realizar diagnósticos diferenciados que permitan llevar a cabo intervenciones ajustadas a las necesidades de estos jóvenes (Zúñiga, 2008).

La psicopatía es uno de los constructos que permite comprender cómo un sujeto puede elicitar una conducta transgresora de las normas sociales. Es considerado un trastorno de personalidad que por su gravedad ha suscitado numerosos estudios a nivel mundial (Cleckley, 1941/1976; Forth, Hart $\&$ Hare, 1990; Hare, 2006), la mayor parte de los cuales se ha realizado en varones adultos. En las últimas décadas se han ampliado las investigaciones a mujeres (Rabucco, 2005), adolescentes y niños. El desarrollo de instrumentos de evaluación de psicopatía en niños y adolescentes ha contribuido a la comprensión de la conducta antisocial en este grupo etario (Forth, Kosson \& Hare, 2003; Murrie, Cornell, Kaplan, McConville \& Levy-Elkon, 2004; Salekin, Brannen, Zalot, Leistico \& Neumann, 2006). Sin embargo, es importante considerar que si bien hay mayor conocimiento de la psicopatía en adultos, es imperioso investigar si este conocimiento es efectivamente aplicable a los adolescentes, si existen diferencias en su manifestación durante la juventud y si se observa estabilidad en los rasgos psicopáticos de los adolescentes o se modifican con el desarrollo evolutivo (Lee, Salekin \& Iselin, 2010).

Por otra parte, es imprescindible revisar los factores de riesgo con el fin de evaluar si son comunes con los de otros trastornos de personalidad, como por ejemplo el Trastorno de Personalidad Antisocial. Generar investigaciones que permitan conocer cómo se comporta el constructo de psicopatía aplicado en la juventud, podría tener implicaciones serias en aspectos legales y en la intervención psicológica en el país, tal como los ha tenido en otros países (Cauffman, Kimonis, Dmitrieva \& Monahan, 2009).

Este artículo tiene como objetivo hacer una revisión teórica de la investigación realizada por Zúñiga (2008) que pretende ser un aporte a la caracterización psicométrica del instrumento Psychopathy Checklist Youth Version, evaluando su confiabilidad y validez; y del estudio de León, Asún y Folino (2010) que contribuye con hallazgos empíricos significativos referentes a factores de riesgo en el desarrollo de un trastorno psicopático en la adultez, cuyo resultado permite tomar las precauciones pertinentes cuando este constructo se evalúa en jóvenes menores de 18 años.

\section{Antecedentes}

\section{Psicopatía}

En la actualidad, la psicopatía ha sido descrita como un constructo multidimensional (Gacono \& Hutton, 1994; Templeman \& Wong, 1994), y constituye un grave trastorno de personalidad caracterizado por alteraciones en cuatro áreas de la personalidad: afectiva, interpersonal, conductual y conducta antisocial (Hare, 1993). Su etiología se asienta en la biología y en la modulación ambiental (Hare, 2007) y se hace énfasis en su diferenciación del Trastorno de Personalidad Antisocial establecido por el DSM-IV (American Psychiatric Association [APA], 1994). Cabe aclarar que si bien serían entidades nosológicas similares con alta comorbilidad, no se trataría de la misma patología (Hare, 2003; Hart \& Hare, 1996).

Theodore Millon (2006) define el Trastorno de Personalidad Antisocial como un patrón complejo de características psicológicas profundamente arraigadas y de difícil modificación que se expresarían en prácticamente todas las áreas conductuales del individuo, caracterizado por una forma independiente o autónoma de construirse a sí mismo y por la conducta antisocial (Millon \& Davis, 1998). Se trataría de sujetos explosivos que se gratifican al dominar y humillar a los demás, temerarios y sin miedo al castigo (Millon, 2006). Su teorización del trastorno de personalidad antisocial se plasma 
en el DSM-III-R y posteriormente en el DSM-IV (American Psychiatric Association [APA], 1987, 1994). Entre los conceptos de psicopatía y trastorno de personalidad antisocial existiría una fuerte relación: los sujetos elaborarían de forma idiosincrásica los eventos, dificultando la incorporación de la norma social (Zúñiga, 2008). Ambos trastornos se manifestarían en conductas transgresoras de los límites personales y/o sociales por parte de los sujetos, acompañados de un estilo de vida antisocial. Las diferencias entre el diagnóstico de psicopatía y trastorno de personalidad antisocial se establece empíricamente y se observa en características afectivas y de la calidad de los vínculos e interrelaciones que el sujeto entabla con los demás.

\section{Adolescencia y psicopatía}

La adolescencia constituye una etapa de búsqueda de sentido de sí mismo y del mundo con base en las operaciones psicológicas que resultan en la producción de pensamientos e ideales propios asentados en experiencias individuales o compartidas (Zúñiga, 2008). Es durante este proceso en el que los jóvenes manifiestan conductas disruptivas que podrían o no tener carácter psicopático. No obstante, es llamativo que a pesar de la prolijidad de los estudios de diversos autores, entre ellos Cleckley (1941/1976), las referencias respecto de las características del trastorno en la niñez o adolescencia son notablemente incompletas y estarían marcadas por las mismas características de la psicopatía en adultos, por lo que se deduce una posible estabilidad de los rasgos psicopáticos. Las investigaciones en niños y adolescentes con características psicopáticas, rasgos de crueldad, narcisismo y conducta impulsiva, indican que estos tienden a manifestar altos niveles de agresividad (Barry, Barry, Deming \& Lochman, 2008), propensión a la búsqueda de excitación y una forma persistente de conducta antisocial similar a los patrones antisociales observados en los adultos. Aun así, en el presente, los investigadores muestran cautela en considerar como persistentes y estables características psicopáticas evaluadas en jóvenes, haciendo énfasis en una disminución de la impulsividad a medida que el adolescente adquiere un sistema de reflexión más desarrollado (Lee et al., 2009). Blonigen, Hicks, Krueger, Patrick y Iacono (2006) plantean que rasgos relacionados a la psicopatía adolescente como la temeridad y la impulsividad antisocial han mostrado estabilidad durante la adolescencia, pero que se modifican con el paso de la adolescencia a la adultez. Harpur y Hare (1994) señalan que las características psicopáticas varían en función de la edad, pero que los componentes afectivos del constructo se mantendrían estables a lo largo de la vida con una disminución de la conducta antisocial.

Cauffman enfatiza la necesidad de la precaución en el uso de los instrumentos cuando se refiere a la evaluación de riesgo de reincidencia en el caso de los jóvenes (Cauffman et al., 2009), en particular en la evaluación de rasgos como la tendencia a la impulsividad que constituye uno de los elementos que ha recibido mayor atención en los estudios con adolescentes (Forth et al., 2003; Harper \& Hare, 1994; Lee et al., 2009).

\section{Evaluación de psicopatía en la adolescencia}

Basándose en los postulados de Cleckley (1941/1976), Robert Hare elabora la Psychopathy Checklist (PCL) para la evaluación de la psicopatía en adultos. Numerosas investigaciones llevaron a su revisión (1991) y a la posterior publicación de la Psychopathy Checklist - Revised (PCL-R) (Hare, 1999, 2003). En Chile se ha realizado una serie de estudios que avalan las características psicométricas del instrumento y su correspondencia con investigaciones internacionales (León, 2009), a la vez que han mostrado su validez y confiabilidad en el medio local (Abalos, Esquivel \& Gallardo, 2004; León et al., 2010).

La presencia de rasgos similares a la psicopatía adulta en sujetos jóvenes y las diferencias en el desarrollo de las personas motivaron a Forth et al. (2003) al desarrollo de la PCL-YV, con el propósito de adaptar el instrumento a la evaluación de las características propias de la adolescencia. Desde su elaboración, son múltiples las investigaciones empíricas que lo respaldan (Forth et al., 2003; Salekin et al., 2006; Salekin \& Debus, 2008; Zúñiga, 2008). 
Otro instrumento que cuenta con investigaciones empíricas que avalan su uso es el Inventario Clínico para Adolescentes de Millon (Millon Clinical Multiaxial Inventory [MACI]), que intenta dimensionar y evaluar la personalidad adolescente en tres ámbitos, integrados en las siguientes escalas clínicas: Patrones de la Personalidad (12 escalas), Preocupaciones Expresadas (8 escalas) y Síndromes Clínicos (7 escalas). En Chile, el MACI ha demostrado adecuados niveles de confiabilidad para muestras normales y de adolescentes con desadaptación social (Vinet \& Alarcón, 2003). Además, han sido creadas y aplicadas dos escalas derivadas para la evaluación de características psicopáticas en la adolescencia (Zúñiga, Vinet \& León, 2011): la Psychopathy Content Scale - MC-20 (Murrie \& Cornell, 2000) y el P-16 (Salekin, Ziegler, Larrea, Anthony \& Bennett, 2003).

\section{Método}

El estudio realizado por León, Asún y Folino (León, 2009) se realizó en una muestra de 293 sujetos varones seleccionados del total de sujetos judicializados y penalizados en los recintos de la Gendarmería de Chile. Los casos fueron clasificados por el personal del área técnica de dicho organismo, que dividió a los sujetos en dos grupos según diagnóstico clínico: psicópatas y no psicópatas. Posteriormente, fueron entrevistados a ciegas por la investigadora y se les aplicó la PCL-R. Se contó con las fuentes de información colateral necesaria para cumplir con los requisitos de aplicación de la prueba. Todas las entrevistas fueron registradas en video y se contó con la autorización informada de los participantes. La correlación ítem-total se realizó por medio de la $r$ de Pearson y se obtuvo el índice kappa para analizar la validación por criterio externo.

Un segundo estudio en curso en el presente, se llevó a cabo con una muestra de 209 sujetos condenados y alojados en el Centro de Detención Penal de Los Andes en Chile entre octubre de 2009 y diciembre de 2010. Se aplicó la PCL-R a la totalidad de los sujetos y se obtuvieron puntajes de consenso y el índice coeficiente de correlación interno entre dos evaluadores. Al igual que en el estudio anterior, las entrevistas se registraron en forma audiovisual y se solicitó un consentimiento informado a los participantes. En ambos estudios se evaluaron las trayectorias de los sujetos y en el segundo, el estado.

En el tercer estudio mencionado en la revisión correspondiente a Zúñiga (2008), la población la conformaron 30 adolescentes varones judicializados en la Región de los Ríos (Chile), seleccionados por medio de un muestreo no probabilístico de tipo intencional. Las edades de los adolescentes estuvieron entre 12 y 17 años. A todos se les aplicó el MACI y la PCL-YV en entrevista videorregistrada con consentimiento informado. Para la evaluación de la consistencia interna de la PCLYV se utilizó el alfa de Cronbach y para evaluar la validez concurrente con el MACI, la $r$ de Pearson. Además, y de forma exploratoria, fueron aplicadas dos escalas derivadas del MACI creadas para la evaluación de características psicopáticas en la adolescencia: la MC-20 (Murrie \& Cornell, 2000) y el P-16 (Salekin et al., 2003).

\section{Resultados}

En la investigación realizada por León et al. (2010) con la PCL-R (Tabla 1), se observa que todos los ítems muestran correlación con el valor total, pudiéndose plantear que cada uno de ellos contribuye notablemente al puntaje total del test. Sin embargo, se destaca que no todos contribuyen con igual peso. Los ítems que tienen las menores correlaciones son: el ítem 18, delincuencia juvenil; el ítem 17, múltiples relaciones maritales breves y el 14, impulsividad. Es importante señalar que el valor del ítem delincuencia juvenil probablemente se vea alterado por la carencia de registros institucionales; para su puntuación se utilizó mayoritariamente la información proporcionada por los mismos sujetos.

En lo referente a la correlación ítem-test la PCLR mostró una excelente correlación en cada uno de sus factores. En la Tabla 2 se observa que las correlaciones de cada factor con el test en su totalidad son altamente significativas. 


\section{TABLA 1}

Estadísticos descriptivos y correlación ítem-total en la investigación realizada en el 2010 por León, Asún y Folino, con la PCL-R.

\begin{tabular}{clccccccc}
\hline \multicolumn{1}{c}{ Ítem } & Nombre de ítem & 0 & 1 & 2 & Omitidos & $M$ & DE & $r$ \\
\hline 1 & Locuacidad y encanto superficial & 74 & 115 & 100 & 4 & 0.96 & 0.756 & $0.690^{* *}$ \\
2 & Grandiosidad y egocentrismo & 64 & 103 & 123 & 3 & 1.07 & 0.792 & $0.648^{* *}$ \\
3 & Necesidad de estimulación y/o tendencia al aburrimiento & 34 & 133 & 120 & 6 & 1.28 & 0.675 & $0.608^{* *}$ \\
4 & Mentira patológica & 97 & 98 & 97 & 1 & 0.82 & 0.797 & $0.674^{* *}$ \\
5 & Nec. de control y manipulac. & 33 & 104 & 155 & 1 & 1.30 & 0.700 & $0.742^{* *}$ \\
6 & Falta de remordimiento y culpa & 10 & 99 & 183 & 1 & 1.50 & 0.570 & $0.686^{* *}$ \\
7 & Superficialidad afectiva & 76 & 113 & 102 & 2 & 0.94 & 0.765 & $0.777^{* *}$ \\
8 & Insensibilidad y falta de empatía & 11 & 144 & 138 & 0 & 1.31 & 0.539 & $0.785^{* *}$ \\
9 & Estilo de vida parasitario & 77 & 178 & 37 & 1 & 0.82 & 0.584 & $0.574^{* *}$ \\
10 & Mal control conductual & 29 & 109 & 154 & 1 & 1.39 & 0.710 & $0.550^{* *}$ \\
11 & Promiscuidad sexual & 97 & 97 & 78 & 21 & 0.89 & 0.792 & $0.643^{* *}$ \\
12 & Problemas de conducta precoces & 98 & 93 & 81 & 21 & 0.93 & 0.833 & $0.606^{* *}$ \\
13 & Falta de metas L.P. realistas & 56 & 135 & 94 & 8 & 1.05 & 0.731 & $0.606^{* *}$ \\
14 & Impulsividad & 4 & 127 & 161 & 1 & 1.56 & 0.527 & $0.259^{* *}$ \\
15 & Irresponsabilidad & 19 & 139 & 124 & 11 & 1.32 & 0.629 & $0.548^{* *}$ \\
16 & Incapacidad para reconocer la responsabilidad propia & 9 & 114 & 170 & 2 & 1.47 & 0.582 & $0.630^{* *}$ \\
17 & Múltiples relaciones maritales breves & 212 & 33 & 28 & 20 & 0.36 & 0.694 & $0.264^{* *}$ \\
18 & Delincuencia juvenil & 95 & 147 & 41 & 10 & 0.84 & 0.678 & $0.393^{* *}$ \\
19 & Quebrantamiento de beneficios al ML o de libertad condicional & 47 & 45 & 108 & 93 & 1.23 & 0.840 & $0.501^{* *}$ \\
20 & Versatilidad Criminal & 103 & 109 & 49 & 0 & 0.69 & 0.733 & $0.670^{* *}$ \\
\hline
\end{tabular}

Nota. ** $\mathrm{p}<0.01$ (dos colas).

Las correlaciones fueron calculadas con base en diferentes $N$ debido a los ítems omitidos. $M=$ Media; $D E=$ Desviación estándar. Fuente elaboración propia.

\section{TABLA 2}

Correlación factor-PCL-R Total

\begin{tabular}{lc}
\hline \multicolumn{1}{c}{ Factor } & $r$ \\
\hline Factor 1 Interpersonal: Ítems 1, 2, 4, 5 & $0.97^{* *}$ \\
Factor 2 Afectivo: Ítems 6, 7, 8, 16 & $0.75^{* *}$ \\
Factor 3 Conductual: Ítems 3, 9, 13, 14, 15 & $0.54^{* *}$ \\
Factor 4 Antisocial: Ítems 10, 12, 18, 19, 20 & $0.40^{* *}$ \\
\hline
\end{tabular}

$r=$ Correlación de Pearson; ** $\mathrm{p}<0.01$ (dos colas).

Fuente elaboración propia.

En relación con el análisis de validación por criterio externo, los resultados permiten sostener que la versión chilena de la Hare PCL-R tiene validez de criterio, contemplando como regla de oro la evaluación clínica realizada por personal penitenciario experimentado y conocedor de los casos. El valor kappa fue alto y estadísticamente significativo. Se destaca que la mayor parte de los casos sin acuerdo se ubicaron entre los que fueron diagnosticados como psicópatas desde la opinión clínica y como no psicópatas con la Hare PCL-R (Tabla 3).

En cuanto a la investigación llevada a cabo por Zúñiga (2008), los estadísticos descriptivos y de confiabilidad por consistencia interna se observan en la Tabla 4.

Zúñiga (2008) estableció para Chile correlaciones entre la prueba de Millon y la PCL-YV de Forth et al. (2003), con correlaciones significativas en las escalas de Tendencia al Abuso de Sustancias $(r=0.47)$, Transgresor $(r=0.43)$, Sumiso $(r=-0.42)$, Predisposición a la Delincuencia $(r=0.41)$, Poderoso $(r=0.38)$, Tendencia a la Impulsividad $(r=0.34)$ e Insensibilidad Social $(r=0.29)$. 
TABLA 3

Diagnóstico de psicopatía según PCL-R y opinión clínica

\begin{tabular}{lccc}
\hline & \multicolumn{2}{c}{ Psicópata según opinión clínica } & \\
\cline { 2 - 3 } & No psicópata & Psicópata & Total \\
\hline No psicópata $(<30)$ & 159 & 26 & 185 \\
\multirow{2}{*}{ Psicópata $(>29)$} & $85.9 \%$ & $14.1 \%$ & $100.0 \%$ \\
& 2 & 106 & 108 \\
\hline Total & $1.9 \%$ & $98.1 \%$ & $100.0 \%$ \\
\hline
\end{tabular}

Kappa $=0.804 ; p=0.001$.

Fuente elaboración propia.

TABLA 4

Estadísticos descriptivos y valores de confiabilidad por consistencia interna de la PCL-YV

\begin{tabular}{clccccc}
\hline \multicolumn{1}{c}{ Facetas } & & & & \multicolumn{2}{c}{ alfa de Cronbach } \\
\hline № & \multicolumn{1}{c}{ Nombre } & $\begin{array}{c}\text { N.o de } \\
\text { Elementos }\end{array}$ & $\mathrm{M}$ & $\mathrm{DE}$ & $\begin{array}{c}\text { Puntuación } \\
\text { Directa }\end{array}$ & $\begin{array}{c}\text { Elementos } \\
\text { Tipificados }\end{array}$ \\
\hline 1 & Interpersonal & 4 & 4.71 & 1.8 & 0.76 & 0.76 \\
2 & Afectivo & 4 & 4.03 & 1.87 & 0.65 & 0.66 \\
3 & Estilo de Vida & 5 & 6.2 & 1.92 & 0.7 & 0.73 \\
4 & Conducta Antisocial & 5 & 6.04 & 2.03 & 0.53 & 0.62 \\
\hline
\end{tabular}

$\mathrm{M}=$ Media; $\mathrm{DE}=$ Desviación estándar.

Fuente elaboración propia.

Además, se correlacionaron exploratoriamente la PCL-YV con las escalas de psicopatía PCS (Murrie \& Cornell, 2000) y la P-16 (Salekin et al., 2003) derivadas del MACI. La PCS correlacionó de forma positiva moderada y altamente significativa con el puntaje total del PCL-YV. Esta relación se focaliza en las Facetas Estilo de Vida y Conducta Antisocial, siendo con esta ultima la relación altamente significativa.

Por su parte la escala P-16 no muestra una correlación importante con el puntaje total del PCL-YV, sin embargo, se observa una correlación positiva moderada con la Faceta Antisocial (Zúñiga et al., 2011).

En relación con los elementos de riesgo evaluados en ambos estudios de León (León et al., 2009; León et al., 2010), el primero (León, 2009; León et al., 2010) puso de manifiesto que los psicópatas tenían más riesgo que los no psicópatas de haber vivido en hogares sustitutos y tenido un comienzo delictivo temprano (Tabla 5), donde se observan diferencias significativas entre psicópatas y no psicópatas.

En un estudio en elaboración (León, 2010) se verifica nuevamente el mayor riesgo medioambiental en la niñez y adolescencia e inicio precoz de la actividad delictiva (Tabla 6). En el segundo estudio (León \& Folino, 2011) se observa una reiteración de los datos.

Este estudio mostró que los sujetos diagnosticados como psicópatas inician la vida delictual en forma más precoz ( $M=13$, psicópatas; no psicópatas, $M=16)$. Esta brecha se habría ampliado de los 14 a los 13 años para psicópatas respecto del primer estudio realizado en el año 2009 (León et al., 2010). En relación con la familia delictual, llama la atención que solo el $39.6 \%$ de las familias de los sujetos diagnosticados como psicópatas, no han tenido problemas con la ley.

En ambos estudios es de anotar que una gran cantidad de los sujetos en centros de detención 
TABLA 5

Factores de riesgo medioambiental en psicópatas y no psicópatas

\begin{tabular}{lcccc}
\hline & Psicópatas & No psicópatas & $\chi 2$ & Significancia \\
\hline Hogares con VIF & $46.3 \%$ & $41.9 \%$ & 3.6 & 0.546 \\
Hogares sustitutos & $58 \%$ & $48.4 \%$ & 5.8 & 0.016 \\
Familia Delictual & $42 \%$ & $35.6 \%$ & 0.9 & 0.355 \\
Delincuencia juvenil & $84 \%$ & $50.5 \%$ & 46.8 & 0.000 \\
Media de edad de inicio delictual & 13 años & 14 años & & \\
\hline
\end{tabular}

Nota. $N=293$

$\mathrm{VIF}=$ Violencia intrafamiliar

Fuente elaboración propia.

TABLA 6

Factores de riesgo medioambiental en psicópatas y no psicópatas

\begin{tabular}{lcccc}
\hline & Psicópatas & No Psicópatas & $\chi 2$ & Significancia \\
\hline Hogares con VIF & $60.7 \%$ & $47.7 \%$ & 1.5 & 0.466 \\
Hogares sustitutos & $78.6 \%$ & $50.3 \%$ & 7.7 & 0.006 \\
Familia delictual & $60.4 \%$ & $48.6 \%$ & 1.4 & 0.499 \\
Delincuencia juvenil & $73 \%$ & $56 \%$ & 13.0 & 0.001 \\
Edad de inicio delictual & Media 14 años & Media 11 años & & \\
\hline
\end{tabular}

Nota. $N=206$

$\mathrm{VIF}=$ Violencia intrafamiliar

Fuente elaboración propia.

penal ha vivido alguna etapa de su infancia en hogares sustitutos, ya sea del Estado o de otros miembros de la familia designados por los entonces tribunales de menores (51.5 \%; $52.6 \%$ ) (León, 2009; León et al., 2010), y que la cifra es mayor en sujetos con diagnóstico de psicopatía en comparación con los no psicópatas (58 \% vs. $48.4 \%$ ) y (78.6 \% vs. $50.3 \%$ ) (León, 2009; León, 2010; León et al., 2010). El estudio realizado por León et al. (2010) demostró en su análisis factorial que el haber vivido en familias con violencia intrafamiliar, el tener familia delictual y el haber convivido en hogares sustitutos del Estado fueron factores que, agrupados en una constelación, correlacionaban significativamente con el Factor 3, Estilo Conductual, y el Factor 4, Estilo Antisocial, en la psicopatía del adulto $(r=0.3 ; r=0.49)$.

Otro factor que llama la atención es que los sujetos diagnosticados como psicópatas tienden a tener en mayor número algún familiar cercano en el mundo delictual (60.4%) (León, 2009; León, 2010). Al descomponer esta cifra encontramos que en los casos de sujetos psicopáticos la madre se ha visto involucrada en delitos en un $14.3 \%$ comparado con un $5.5 \%$ de los no psicópatas. La mayor parte de las veces constituyen familias delictuales y el delito es el narcotráfico (León, 2010).

\section{Discusión}

Los datos obtenidos en los estudios mencionados indican que los tests PCL-R y PCL-YV son instrumentos válidos y confiables para ser usados el primero en adultos y el segundo en adolescentes. Ambos métodos de evaluación mostraron buena consistencia interna y adecuada validez por criterio externo. Sin embargo, es importante destacar que la falta de coincidencia diagnóstica se evidencia en los falsos positivos cuando es utilizado el diagnóstico clínico con base en el DSM-IV. Esto constituye un punto preocupante en el diagnóstico de psicopatía adulta, más aún en el diagnóstico de psicopatía en jóvenes. 
En el estudio en curso (León \& Folino, 2011) se evalúa la prevalencia del trastorno psicopático y del trastorno de personalidad antisocial, y las cifras preliminares muestran una concordancia con estudios a nivel mundial (13\% para la psicopatía y $67 \%$ en el caso del trastorno de personalidad antisocial).

La evaluación de personalidades transgresoras como son los casos de Trastorno de Personalidad Antisocial y la psicopatía cobra especial importancia debido a que plantean el inicio precoz entre sus criterios. El DSM-IV establece como criterio que los trastornos conductuales se hayan iniciado antes de los 15 años (APA, 1994) y el trastorno psicopático, tal como lo señala Hare, implica la existencia de perturbaciones conductuales severas antes de los 12 años. Las características personales en la infancia y la adolescencia son particularmente maleables por los elementos ecoculturales y pueden estar asociadas con diversas influencias en la crianza. En esta línea se adicionan los hallazgos locales de influencias negativas, detectando un mayor riesgo en los sujetos que cumplen penas en centros de reclusión. Es especialmente llamativa la asociación existente entre psicopatía y el hecho de haber vivido en hogares sustitutos del Estado. Por ende, los hallazgos estimulan la reflexión sobre cómo generar factores protectores en la población que, durante su infancia y adolescencia, se ve expuesta a familias delictuales, con violencia intrafamiliar o con experiencias de vida en hogares sustitutos.

De esta manera, los estudios realizados no solo aportan dos instrumentos altamente confiables, sino que además entregan datos a los que hay que prestar especial atención sobre todo por parte de los organismos del Estado que cumplen con el mandato social de protección a la infancia vulnerable. Cuando un joven es enviado a un hogar sustituto del estado, con la idea de otorgarle protección, es fundamental preguntarse antes qué impacto y qué consecuencias tendrá esa medida en su desarrollo. Los factores de riesgo dados a conocer en el presente artículo permiten reflexionar acerca de la calidad con que nuestra sociedad está cumpliendo el mandato de protección a su infancia, y si las medidas son realmente basadas en evidencia científica.

Es fundamental mantener una actitud de cautela en la aplicación del constructo psicopático en la etapa juvenil. Algunos estudios refieren correlaciones débiles entre rasgos psicopáticos y reincidencia violenta (Cauffman et al., 2009; Douglas, Webster, Eaves, Wintrup \& Hart, 1996; Edens, Skeem, Cruise \& Cauffman, 2001; Hart, Hare, Forth, Monahan \& Steadman, 1994). Además, en la evaluación del adolescente es relevante contemplar que algunas características psicopáticas no se estabilizarían hasta la adolescencia tardía o el inicio de la adultez (Seagrave \& Grisso, 2002), y que la utilidad predictiva de la psicopatía en adolescentes podría limitarse al corto plazo (Edens et al., 2001).

Como si todo ello fuera poco, la aplicación de la PCL-YV requiere una acabada formación y el tiempo necesario para realizar una entrevista en profundidad y revisar los antecedentes. De todas maneras, con la cautela científica correspondiente, la presencia de los rasgos debería ser explorada y para ello nada mejor que considerar este instrumento o algún otro de los que hasta el momento han demostrado buenas propiedades psicométricas (Millon, David \& Millon, 1997) para la evaluación de rasgos psicopáticos en los jóvenes.

Si bien es cierto que la aplicación de instrumentos altamente especializados, tal como se mencionó anteriormente, es un proceso delicado que requiere alta formación y uso de uno de los recursos más limitados con los que cuentan las instituciones: el tiempo, también es cierto que las consecuencias de no prestar atención a las cifras entregadas son graves para nuestra sociedad.

Por último, si bien resulta razonable considerar los rasgos en cuestión dentro de los menos accesibles al tratamiento, nada justifica excluirlos de esta posibilidad (Forth \& Kosson, 2005), sino, por el contrario, todo orienta a fortalecer los recursos de intervención específicos y que estos sean proporcionados de modo pertinente.

Finalmente, si bien la debilidad del primer estudio, muestra intencionada, queda superada 
por el segundo estudio de León al tomar como muestra el universo de los sujetos, lejos está de haberse acabado la investigación en la temática de psicopatía, ya que la heurística aconseja estudios con mayor amplitud muestral tanto en jóvenes como en adultos y la focalización en los aspectos que surgen como hallazgos, de modo de establecer con claridad los factores de riesgo para adolescentes chilenos tanto en evaluación de psicopatía como en la de trastorno de personalidad antisocial.

\section{Referencias}

Abalos, C., Esquivel, N. \& Gallardo, C. (2004). Adaptación de la Escala de Clasificación de PsicopatíaRevisada (PCL-R) de R. Hare en población reclusa del Centro de Detención Preventiva de San Miguel. Memoria para optar al título de Psicólogo, Universidad de Chile, Santiago. Disponible en http:// www.cybertesis.cl/tesis/uchile/2004/abalos_c/sources/abalos_c.pdf

American Psychiatric Association. (1987). Manual diagnóstico y estadístico de los trastornos mentales (3.. - ed. rev.). Barcelona: Masson.

American Psychiatric Association. (1994). Diagnostic and statistical manual of mental disorders (4. ${ }^{\mathrm{a}}$ ed.). Washington, DC: Autor.

Barry, T. D., Barry, C. T., Deming, A. M. \& Lochman, J. E. (2008). Stability of psychopathic characteristics in childhood: The influence of social relationship. Criminal Justice and Behavior, 35(2), 244-262. doi: 10.1177/0093854807310508

Blanco, J. (2010, agosto). Violencia y delincuencia juvenil: desafíos en relación con los programas y respuestas del sistema. Trabajo presentado en el Seminario Derechos de los Jóvenes, Justicia y Seguridad Ciudadana, Santiago de Chile. Disponible en http://www. pazciudadana.cl/docs/pub_20101007152712.pdf

Blonigen, D. M., Hicks, B. M., Krueger, R. F., Patrick, C. J. \& Iacono, W. G. (2006). Continuity and change in psychopathic traits as measured via normalrange personality: A longitudinal-biometric study. Journal of Abnormal Psychology, 115(1), 85-95. doi: 2006-02317-010 [pii] 10.1037/0021-843X.115.1.85

Cauffman, E., Kimonis, E. R., Dmitrieva, J. \& Monahan, K. C. (2009). A multimethod assessment of juve- nile psychopathy: Comparing the predictive utility of the PCL:YV, YPI, and NEO PRI. Psychological Assessment, 21(4), 528-542. doi: 2009-22537-008 [pii] 10.1037/a0017367

Cleckley, H. (1941/1976). The mask of sanity (5 ${ }^{\text {th }}$. ed.). St. Louis, MO: Mosby.

Douglas, K. S., Webster, C. D., Eaves, D., Wintrup, A. \& Hart, S. D. (1996, marzo). A new scheme for the asessment of dangerousness and the prediction of violence. Trabajo presentado en la Biennal Meeting of the American Psychology-Law Society, Hilton Head, South Carolina, EE. UU.

Edens, J. F., Skeem, J. L., Cruise, K. R. \& Cauffman, E. (2001). Asessment of juvenile psychopathy and its association with violence: A critical review. WileyInterScience, 19(1), 53-80.

Forth, A. E., Hart, S. D. \& Hare, R. D. (1990). Assessment of psychopathy in male young offenders. Psychological Assessment: A Journal of Consulting and Clinical Psychology, 2(3), 342-344.

Forth, A. \& Kosson, D. S. (2005). Criminal recidivism in juvenile. En Group DR (Ed.), The application of the PCL-YV. Chicago: Eleanor Roosevelt University Hospital.

Forth, A., Kosson, D. \& Hare, R. D. (2003). The Hare Psychopathy Checklist: Youth Version. Technical Manual. New York/Toronto: Multi-Health System, Inc.

Gacono, C. B. \& Hutton, H. E. (1994). Suggestions for the clinical and forensic use of the Hare Psychopathy Checklist-Revised (PCL-R). International Journal of Law and Psychiatry, 17(3), 303-317. doi: 0160-2527(94)90032-9 [pii]

Hare, R. D. (1993). Without conscience: The disturbing world of the psychopaths among us. New York: Simon \& Schuster.

Hare, R. (1999). Psychopathy as a risk for violence. Psyquiatric Quarterly, 70(3), 181-197.

Hare, R. (2003). The Hare Psychopathy Checklist. Revised manual (2nd. ed.). Toronto: Multi-Health Systems, Inc.

Hare, R. (2006). The PCL-R and the evaluation of psychopathy. La Psicopatía iEs posible su evaluación? Santiago de Chile: Pontificia Universidad Católica.

Hare, R. (2007, marzo). The dark side of personality. E. Punsett (Coord.), Emociones básicas: el impacto de 
la ciencia en la sociedad. Madrid: Fundación Central Hispana de Santander.

Hare, R. D., Hart, S. D. \& Harpur, T. J. (1991). Psychopathy and the DSM-IV criteria for antisocial personality disorder. Journal of Abnormal Psychology, 100 (3), 391-398.

Harpur, T. J. \& Hare, R. D. (1994). Assessment of psychopathy as a function of age. Journal of $A b$ normal Psychology, 103(4), 604-609.

Hart, S. D. \& Hare, R. D. (1996). Psychopathy and antisocial personality disorder. Current Opinion in Psychiatry, 9(2), 129-132.

Hart, S. D., Hare, R. D., Forth, A. E., Monahan, J. \& Steadman, H. J. (1994). Psychopathy as a risk marker for violence: Development and validation of a screening version of the Revised Psychopathy Checklist. En J. Monahan \& H. Steadman (Eds.), Violence and mental disorder: Developments in risk assessment (pp. 81-98). Chicago, IL: University of Chicago Press.

Lee, Z., Salekin, R. \& Iselin, A. M. (2010). Psychopathic traits in youth: Is there evidence for primary and secondary subtypes? Journal of Abnormal Child Psychology, 38(3), 381-393.

León, E. (2009). Determinación del nivel de fiabilidad y validez del PCL-R en población forense masculina chilena. Tesis de Maestría en Psicología Social con mención en Psicología Jurídica, Universidad de Valparaíso, Chile.

León, E. (2010). La psicopatía en prisioneros chilenos: prevalencia y métodos de evaluación. Tesis de Doctorado, Universidad Nacional de La Plata, Buenos Aires.

León, E., Asún, D. \& Folino, J. (2010). Confiabilidad y validez de la versión chilena de la Hare PCL-R. Revista de la Facultad de Medicina, 58(2), 103-114.

León, E. \& Folino, J. (2011). Estados y trayectorias desadaptativas relevantes en la planificación asistencial en las cárceles. Tercera Época, 2(3), 1-2.

Millon, T. (2006). Millon Clinical Multiaxial Inventory-III (MCMI-III) manual. Minneapolis, MN: Pearson Assessments.

Millon, T. \& David, R. D. (1998). Ten subtypes of psychopathy. En T. Millon, E. Simonsen, M. BirketSmith \& R. D. Davis (Eds.), Psycopathy: Antisocial
Criminal and violent behavior (pp. 161-170). New York: Guilford Press.

Millon, T., David, R. D. \& Millon, C. (1997). Millon Clinical Multiaxial Inventory III (2 ${ }^{\text {nd }}$. ed.). Minneapolis, MN: National Computer Systems.

Murrie, D. \& Cornell, D. (2000). The Millon Adolescent Clinical Inventory and psychopathy. Journal of Personality Assessment, 75(1), 110-125.

Murrie, D. C., Cornell, D. G., Kaplan, S., McConville, D. \& Levy-Elkon, A. (2004). Psychopathy scores and violence among juvenile offenders: A multimeasure study. Behavioral Sciences \& the Law, 22(1), 49-67. doi: 10.1002/bsl.573

Rabucco, D. (2005). Validación empírica de la Escala de Calificación de Psicopatía Revisada (PCL-R) de $R$. Hare en la población penal de la provincia de Valparaíso. Tesis para Licenciado en Psicología, Universidad del Mar, Valparaíso, Chile.

Salekin, R. T., Brannen, D. N., Zalot, A. A., Leistico, A. -M. \& Neumann, C. S. (2006). Factor structure of psychopathy in youth. Criminal Justice and Behavior, 33(2), 135-157. doi: 10.1177/0093854805284416

Salekin, R. \& Debus, S. (2008). Assessing child and adolescent psychopathy. En R. Jackson (Ed.), Learning forensic assessment (pp. 347-383). New York: Routledge.

Salekin, R., Ziegler, T., Larrea, M., Anthony, V. \& Bennett, A. (2003). Predicting dangerousness with two Millon Adolescent Clinical Inventory Psychopathy Scales: The importance of egocentric and callous traits. Journal of Personality Assessment, 80(2), 154-163.

Seagrave, D. \& Grisso, T. (2002). Adolescent development and the measurement of juvenile psychopathy. Law and Human Behavior, 26(2), 219-239.

Templeman, R. \& Wong, S. (1994). Determining the factor structure of the Psychopathy Checklist: A converging approach. Multivariate Experimental Clinical Research, 10(3), 156-166.

Vinet, E. \& Alarcón, P. (2003). El Inventario Clínico para Adolescentes de Millon (MACI) en la evaluación de adolescentes chilenos. Psykhe, 12(1), 39-55.

Zúñiga, D. P. (2008). Caracterización psicométrica del instrumento Psychopathy Checklist: Youth version (PCL:YV). Tesis para Magíster, Universidad de La Frontera., Temuco, Chile. 
Zúñiga, D., Vinet, E. \& León, E. (2011). Caracterización psicométrica del Psychopathy Checklist: Youth Version (PCL:YV) en adolescentes chilenos. Terapia Psicológica, 29(1), 25-31. 
